\title{
On-chip quantum tomography of mechanical nanoscale oscillators with guided Rydberg atoms
}

\author{
A. Sanz-Mora, ${ }^{1, *}$ S. Wüster, ${ }^{1,2,3}$ and J.-M. Rost ${ }^{1}$ \\ ${ }^{1}$ Max Planck Institute for the Physics of Complex Systems, Nöthnitzer Strasse 38, 01187 Dresden, Germany \\ ${ }^{2}$ Department of Physics, Bilkent University, 06800 Çankaya, Ankara, Turkey \\ ${ }^{3}$ Department of Physics, Indian Institute of Science Education and Research, Bhopal, Madhya Pradesh 462 023, India
}

(Received 31 March 2017; revised manuscript received 27 June 2017; published 27 July 2017)

\begin{abstract}
Nanomechanical oscillators as well as Rydberg-atomic waveguides hosted on microfabricated chip surfaces hold promise to become pillars of future quantum technologies. In a hybrid platform with both, we show that beams of Rydberg atoms in waveguides can quantum coherently interrogate and manipulate nanomechanical elements, allowing full quantum state tomography. Central to the tomography are quantum nondemolition measurements using the Rydberg atoms as probes. Quantum coherent displacement of the oscillator is also made possible by driving the atoms with external fields while they interact with the oscillator. We numerically demonstrate the feasibility of this fully integrated on-chip control and read-out suite for quantum nanomechanics, taking into account noise and error sources.
\end{abstract}

DOI: 10.1103/PhysRevA.96.013855

\section{INTRODUCTION}

Quantum optomechanics was originally developed in the context of gravitational wave detection [1]. Subsequently, it took up the challenge to cool nanoscale quantum systems all the way to their ground state [2], and more generally to gain the level of control over them that we are used to having over quantum optical systems [3-5], e.g, through coupling to nonclassical light [6,7]. Experiments on the quantum nondemolition (QND) read-out of the phonon state of a nanomechanical oscillator or its state tomography have only begun recently $[8,9]$ and most existing proposals interface the oscillator with a cavity [10-14].

For torsional oscillators [15,16], we develop in the following a scheme without direct cavity interfacing, allowing for integration of mechanical and measurement elements into the same nanofabricated substrate using Rydberg atoms. With their long lifetimes and strong long-range interactions [17], they mingle naturally with the time and spatial scales of optomechanics. Furthermore, with accessible atomic transition frequencies spanning orders of magnitude when varying the principal quantum number $v$, Rydberg atoms can interface to a wide range of nanomechanical elements with different oscillation frequencies [3-5].

Recent advances in manipulation and control of Rydberg atoms through on-chip waveguides [18-20] as well as in retaining atomic coherence closer to chip surfaces [21-24] render Rydberg on-chip integration promising and realistic by matching Rydberg-atom interaction ranges with the spatial $\mu \mathrm{m}$ scales of the chip geometry.

To achieve a full quantum tomography of the torsional nano-oscillator, the Rydberg atoms have to fulfill a twofold role: First, the atomic Rydberg beam in the waveguide passing by the oscillator acts as probe for the oscillator state [25,26]: controlled electrostatic interactions between the oscillator and the atoms cause a phonon-number-dependent phase shift, to be read out interferometrically [27-31]. Second, we can coherently displace the nanomechanical oscillator by

\footnotetext{
*adnsmora@pks.mpg.de
}

externally driving the Rydberg atom while it is passing by the oscillator in order to scan the oscillator state in a controlled fashion. In this step, the Rydberg atom acts as a mediator for quantum control of the oscillator.

So far, destructive Wigner tomography [32] has been proposed, as well as the use of classical oscillators for atomic quantum state control [33], which could also be achieved through interaction of nanomechanical elements with atoms or molecules [34-36].

We extend these works by transferring Rydberg-atombased QND detection developed in the context of cavityQED [27-31] to the realm of quantum nanomechanics, and integrate all these functional elements into a versatile onchip Rydberg-atomic probe technique without the need of a cavity.

The organization of this paper is as follows. In Sec. II we describe an interfacing between a Rydberg-atom waveguide and a torsional oscillator, and propose methods for a QND read-out of mechanical oscillation states and their tomographical reconstruction. Next, we provide the Hamiltonian governing the system dynamics in Sec. III, and numerically assess the feasibility of the proposed QND detection and state tomography schemes subject to noise and decoherence elements in Secs. IV and V, respectively. Finally, we summarize our findings in Sec. VI.

\section{HYBRID SETUP OF NANOTORSIONAL OSCILLATOR AND RYDBERG WAVEGUIDE}

While our scheme is quite general, we nevertheless will consider a carbon nanotube (CNT) as torsional oscillator to be specific. Interfacing the Rydberg waveguide, we will explicitly take into account the expected dominant decoherence sources. The CNT is clamped to a chip surface and equipped with a weight at one end, as, e.g., in [37,38], which allows for tuning the oscillation frequency. The weight will be a ferroelectric nanoparticle with a permanent electric dipole moment $\mathbf{d}_{\mathrm{osc}}$ [39], providing a simple and adjustable interaction between oscillator and Rydberg atoms, independent of surface and material properties. The Rydberg atoms, confined to an atomic 


\section{SYSTEM HAMILTONIAN}

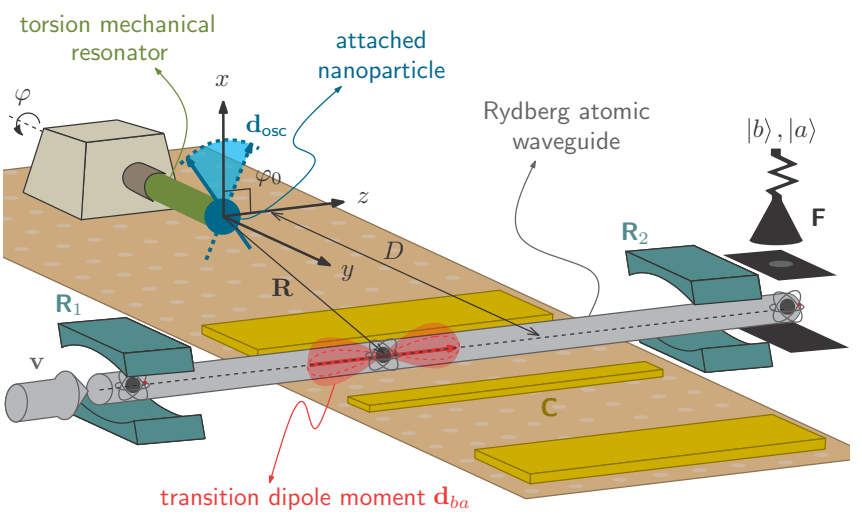

FIG. 1. Schematic of the coupled Rydberg-atomic waveguide (gray) and torsion mechanical oscillator (green). The beam passes the oscillator with impact parameter $D$ and velocity v. Oscillations of the torsion angle $\varphi$ modulate the interaction between the permanent electric moment $\mathbf{d}_{\mathrm{osc}}$ of a ferroelectric load (blue) and the transition dipole moment $\mathbf{d}_{b a}$ involving atomic Rydberg states $|b\rangle$ and $|a\rangle$. While a train of atoms interacts one by one with the oscillator, the states incur a phase shift dependent on the state of the oscillator. This shift is interferometrically read out using microwave $\pi / 2$ pulses in regions $\mathrm{R}_{1 / 2}$ and state detection in $\mathrm{F}$. Quantum coherent manipulation of the oscillator for quantum tomography uses additional external driving of the atoms through a coplanar microwave waveguide in region $\mathrm{C}$.

waveguide $[25,40]$, pass the oscillator with impact parameter $D$ as shown in Fig. 1 .

First, the atoms in the guide are excited to a Rydberg state $|a\rangle$ (not shown in Fig. 1). In the region $\mathrm{R}_{1}$ of Fig. 1 the waveguide passes a microwave cavity, which generates a Rabi $\pi / 2$ pulse tuned to the transition between $|a\rangle$ and a second Rydberg state $|b\rangle$. As a consequence, atoms in the state $[|a\rangle+|b\rangle] / \sqrt{2}$ are produced [41].

Parameters are chosen such that the interactions with the oscillator (in the coupling region $\mathrm{C}$ ) effect only a relative phase shift between $|a\rangle$ and $|b\rangle$, to be inferred from detection of the Rydberg state at $\mathrm{F}$ after a second $\pi / 2$ pulse in region $\mathrm{R}_{2}$. If the oscillator is in a Fock state, such a Ramsey interferometric measurement leaves the oscillator state unchanged, thus furnishing a QND measurement. For more general oscillator states, a series of these measurements will gradually collapse the state towards a phonon number (Fock) state [42]. Repeating such series multiple times eventually reveals the entire phonon-number distribution.

Full quantum state tomography requires knowledge of the phases between different number states, which can be obtained after quantum coherently displacing the oscillator prior to the phonon-number distribution measurement. To obtain a well-defined displacement, we propose to externally drive Rydberg atoms while they pass through the strong interaction region $\mathrm{C}$ as discussed below. For a well-defined coupling, the driving should target only the Rydberg atoms and not directly the oscillator by using well-localized evanescent fields from a coplanar microwave guide $[43,44]$ or a three-photon off-resonant Raman transition [45].
We formalize our scheme with the Hamiltonian

$$
\hat{H}=\hat{H}_{\text {osc }}+\hat{H}_{\text {at }}+\hat{H}_{\text {int }}+\hat{H}_{\text {coup }}
$$

to demonstrate quantitatively the feasibility of this protocol. The oscillator with frequency $\omega_{\mathrm{osc}}$ is described by $\hat{H}_{\mathrm{osc}}=$ $\hbar \omega_{\text {osc }} \hat{c}^{\dagger} \hat{c}$, with corresponding oscillator states $|n\rangle$ and ladder operators $\hat{c}, \hat{c}^{\dagger}$. The Hamiltonian for the internal state of a single atom is $\hat{H}_{\text {at }}=\hbar \omega_{b a} \hat{\sigma}_{b b}$, where $\hat{\sigma}_{\mu^{\prime} \mu}=\left|\mu^{\prime}\right\rangle\langle\mu|$ denotes the atomic transition operator between levels $\left|\mu^{\prime}\right\rangle$ and $|\mu\rangle$, and $\omega_{\mu^{\prime} \mu}$ the corresponding Bohr frequency. Motion of the atoms in the waveguide is treated classically as described below. The atom-oscillator coupling $\hat{H}_{\text {int }}$ is due to electric dipole-dipole interactions between the transition dipole of the atom and the permanent dipole of the nanoparticle attached to the oscillator. By choosing the atomic transition dipole $\mathbf{d}_{b a}=\left\langle b\left|\hat{\mathbf{d}}_{\mathrm{at}}\right| a\right\rangle$ ( $\hat{\mathbf{d}}_{\mathrm{at}}$ is the atomic dipole operator) along the $z$ axis and for an atom precisely at the center of the waveguide, we find (Appendix A 2)

$$
\hat{H}_{\text {int }}=-\hbar \mathcal{K}(\mathbf{R})\left[\hat{c}^{\dagger}+\hat{c}\right]\left[\hat{\sigma}_{b a}+\hat{\sigma}_{a b}\right] .
$$

Here, the interaction strength is $\mathcal{K}(\mathbf{R})=\mathcal{K}_{0} f(\mathbf{R})$, where $\mathcal{K}_{0}=$ $V_{0} / \sqrt{2 \hbar \omega_{\mathrm{osc}} I}$, with $V_{0}=\left\|\mathbf{d}_{b a}\right\|\left\|\mathbf{d}_{\mathrm{osc}}\right\| /\left[4 \pi \varepsilon_{0} D^{3}\right]$, and $I$ the moment of inertia of the oscillator (CNT and nanoparticle). The coordinate vector $\mathbf{R}=(X, Y, Z)$, with $R=\|\mathbf{R}\|$, points from the center of the nanoparticle in equilibrium (origin of our Cartesian coordinate system) to the atom in the waveguide, as shown in Fig. 1. Then, the interaction amplitude becomes $f(\mathbf{R})=[D / R]^{3}\left[1-3 Z^{2} / R^{2}\right]$. We have assumed small excursions of the oscillator from an equilibrium torsional angle $\varphi_{0}=\pi / 2$.

Finally, $\hat{H}_{\text {coup }}=\hbar \Omega(t)\left[\hat{\sigma}_{b a}+\hat{\sigma}_{a b}\right] / 2$ represents the controllable interstate resonant coupling with Rabi frequency $\Omega(t)$ in dipole and rotating wave approximations. This term is due to a microwave field in regions $\mathrm{R}_{1}, \mathrm{R}_{2}$ and possibly $\mathrm{C}$.

\section{SIMULATED QUANTUM NONDEMOLITION MEASUREMENTS OF THE PHONON NUMBER}

We consider a scenario where the atomic transition frequency $\omega_{b a}$ is much closer to resonance with the oscillator $\omega_{\text {osc }}$ than any other transition frequency. This justifies taking into account atomic states $|a\rangle,|b\rangle$ only. There are two advantages in choosing these as Rydberg states: (i) For a wide range of mechanical oscillation frequencies $1 \mathrm{MHz}<\omega_{\text {osc }}<10 \mathrm{GHz}$, some near-resonant atomic transitions can be found with $\omega_{b a}=\omega_{\mathrm{osc}}+\delta$ and atom-oscillator detuning $\delta$ much smaller than energy gaps to any other atomic states. (ii) The large Rydberg transition dipoles $\mathbf{d}_{b a}$ provide strong coupling $\mathcal{K}_{0}$ between atom and oscillator without too close proximity.

Nonetheless, we can achieve a situation regime where the atom and oscillator are far off resonant with respect to the coupling strength $\mathcal{K}(\mathbf{R})$. Then, creation and destruction of phonons through (2) is suppressed, during a detection of a single atom or of many atoms in a series. Thus, while the compound system of atom plus oscillator adiabatically follows its interacting eigenstates as the atom passes the oscillator, the interaction only causes a phase shift $\Phi^{(n)}[\delta]$ between the superimposed states $|a\rangle,|b\rangle$ depending on the detuning $\delta$ and 
the phonon number $n$. After the second Rabi $\pi / 2$ pulse at $R_{2}$, this phase shift affects the results of destructive state measurement in F. Ultimately, after a succession of such measurements we may infer $\cos \left(\Phi^{(n)}[\delta]\right)$. Further details can be found in Appendix B and [46].

We now proceed to simulate measurements, taking into account decoherence sources to explore the practical limitations arising through the vicinity of a microchip surface and the Rydberg atom waveguide. We work in a frame rotating with the oscillator frequency, keeping only resonant terms in (2), and employ the master equation for the density matrix $\hat{\rho}$ of harmonic oscillator plus a single atom $(\hbar=1)$

$$
\dot{\hat{\rho}}=-i[\hat{H}(t), \hat{\rho}]+\sum_{\alpha} \mathcal{L}_{\hat{L}_{\alpha}}[\hat{\rho}] .
$$

The Hamiltonian is time dependent due to the classical (uniform) atomic motion $\mathbf{R}=\mathbf{R}(t)$ (see Appendix B). We include several Lindblad terms $\mathcal{L}_{\hat{O}}[\hat{\rho}]=\hat{O} \hat{\rho} \hat{O}^{\dagger}-\left(\hat{O}^{\dagger} \hat{O} \hat{\rho}+\right.$ $\left.\hat{\rho} \hat{O}^{\dagger} \hat{O}\right) / 2$ accounting for decoherence processes, which are fully described in Appendix D: (i) Mechanical oscillator states decohere because they are coupled to a heat bath at temperature $T_{\text {osc }}$ with mechanical energy damping rate $\Gamma_{\text {osc }} /(2 \pi)=50 \mathrm{~Hz}$ [47]. Atomic Rydberg states (ii) undergo incoherent relaxation between $|a\rangle$ and $|b\rangle$ due to black-body radiation and (iii) dephase with a rate $\Gamma_{\text {deph }} /(2 \pi)=1.5 \mathrm{kHz}$ due to stray electric fields from the oscillator-bearing surface. The latter effect, a major challenge for Rydberg-atom quantum technologies near solid-state surfaces, has been steadily reduced [22,24]. (iv) Finally, we describe the widths of the interrogating atomic beam as a random distribution of initial transverse atom positions with respect to the beam axis as well as a distribution of their coaxial velocities. Trajectories are then explicitly modeled using Eq. (3). We set transverse atomic velocities to zero to account for the confinement of the atoms in the waveguide.

In our simulations, we consider states $|a\rangle=$ $\left|v S_{1 / 2}, m_{J}=\frac{1}{2}\right\rangle$ and $|b\rangle=\left|\nu P_{1 / 2}, m_{J}=\frac{1}{2}\right\rangle$ of ${ }^{87} \mathrm{Rb}$ with principal quantum number $v=80$. Their resonance frequency is $\omega_{b a} /(2 \pi) \simeq 6835.81 \mathrm{MHz}$ with transition dipole moment $\left\|\mathbf{d}_{b a}\right\| \simeq 6711 e a_{0}$ (where $e$ is the electron charge and $a_{0}$ the Bohr radius). A 148.54-nm-long and 75.79-nm-wide CNT with a spherical ferroelectric load can yield a moment of inertia $I \simeq 1.12 \times 10^{-32} \mathrm{~kg}^{2} \mathrm{~m}$ with torsional oscillation frequency $\omega_{\text {osc }} /(2 \pi) \simeq 6848.69 \mathrm{MHz}$ (see Appendix A 1), and thus a small atom-oscillator detuning $\delta /(2 \pi) \simeq 12.88 \mathrm{MHz}$. A dipole of strength $\left\|\mathbf{d}_{\text {osc }}\right\| \simeq 3.04 \times 10^{9} e a_{0}$ can be attached. We choose an impact parameter $D=21.68 \mu \mathrm{m}$, and hence a coupling constant $\mathcal{K}_{0} /(2 \pi)=0.64 \mathrm{MHz}$. The transverse atomic wave-guide widths are $\sigma_{X}=\sigma_{Y}=0.51 \mu \mathrm{m}$. The standard deviation of the coaxial atomic velocity is $\sigma_{v_{Z}}=0.01 \mathrm{~m} \mathrm{~s}^{-1}$.

To illustrate measurements of phonon numbers down in the quantum regime $\left(\omega_{\text {osc }} \gtrsim k_{\mathrm{B}} T_{\text {osc }}\right.$, with $k_{\mathrm{B}}$ the Boltzmann constant) we focus on the range $n=0, \ldots, 5$. Then, the corresponding probabilities $P_{b}$ for the atom to end up in state $|b\rangle$ should be distinguishable, as in the example of Fig. 2(a). The beam impact parameter $D$ and atom velocities can be adjusted to yield phase shifts $\Phi^{(n)}[\delta]$ that fulfill this requirement. The figure demonstrates that even taking into
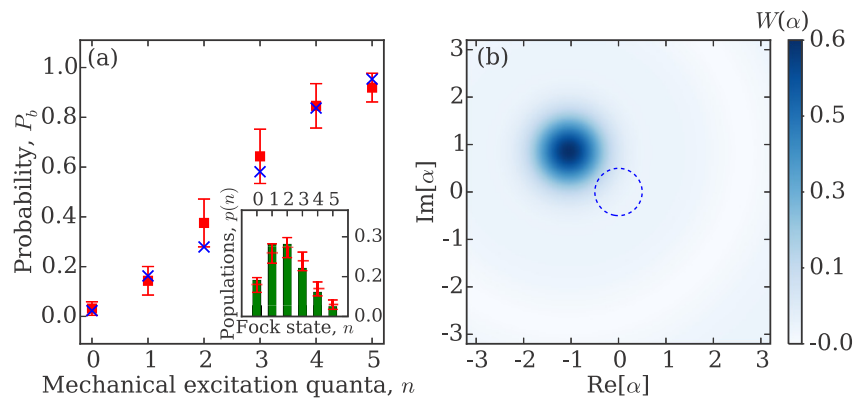

FIG. 2. (a) Phonon-state-dependent probability $P_{b}$ for the probe atom to arrive in state $|b\rangle$. (Blue $\times$ ) Ideal result for an atom at waveguide center, (red box and error bars) average and standard deviation with finite width atomic beam. Both data sets include decoherence. The inset shows the inferred phonon distribution for a coherent oscillator state $|\alpha\rangle$ with coherent amplitude $\alpha=\sqrt{2}$. (b) Wigner function of mechanical oscillator state. Before displacement we take the ground state (contour), after displacement operation $\hat{\mathcal{D}}\left[\alpha_{N}(\tau)\right]$ we obtain a coherent state (color). We use $\alpha_{N}(\tau) /\left|\alpha_{N}(\tau)\right|=$ $-[1-\mathrm{i}] / \sqrt{2}$, with $\left|\alpha_{N}(\tau)\right| \simeq 1.35, \Omega /(2 \pi)=\sqrt{2} / 2 \mathrm{MHz}$ (see text).

account deviations in $\Phi^{(n)}[\delta]$ due to imperfections as discussed above, a clear inference of $|n\rangle$ can be made. For an oscillator in a Fock state a sequence of QND measurements using atoms can yield the probability $P_{b}$. If the initial oscillator state $|\Psi\rangle$ is not a Fock state, this sequence initially quickly collapses it into one, say $|n\rangle$, with probability $p_{n}=|\langle n \mid \Psi\rangle|^{2}$. A series of such collapse sequences, starting from a reinitialized oscillator state $|\Psi\rangle$, then yields the entire phonon distribution $p_{n}$ as shown exemplarily in the inset of Fig. 2(a).

\section{WIGNER TOMOGRAPHY OF A TORSIONAL OSCILLATOR STATE}

Phonon-state QND measurements yield the probabilities $p_{n}=\varrho_{n n}=\operatorname{tr}[|n\rangle\langle n| \hat{\varrho}]$, where $\hat{\varrho}$ is the reduced density matrix for the oscillator, but no coherences between $|n\rangle,|m\rangle$. The full quantum state of the oscillator may be inferred from a tomographical reconstruction of the Wigner function

$$
W(\alpha)=\frac{2}{\pi} \operatorname{tr}\left[\hat{\mathcal{D}}^{\dagger}(\alpha) \hat{\varrho} \hat{\mathcal{D}}(\alpha) \hat{\Pi}\right],
$$

where $\hat{\mathcal{D}}(\alpha)=\exp \left[\alpha \hat{c}^{\dagger}-\alpha^{*} \hat{c}\right]$ is the displacement operator for a complex amplitude $\alpha$ and $\hat{\Pi}=e^{\mathrm{i} \pi \hat{c}^{\dagger} \hat{c}}$ is the phononnumber parity operator. Equation (4) is the expectation value of $\hat{\Pi}$ in the state $\hat{\varrho}(-\alpha)=\hat{\mathcal{D}}(-\alpha) \hat{\varrho} \hat{\mathcal{D}}^{\dagger}(-\alpha)$. We can thus obtain $W(\alpha)$ as $W(\alpha)=[2 / \pi] \sum_{n}(-1)^{n} \tilde{p}_{n}$ from a phonon distribution $\tilde{p}_{n}$ as in Fig. 2(a), after a coherent displacement by $-\alpha$.

An established method for the quantum coherent displacement of nanomechanical oscillators does not yet exist. A major advantage of the on-chip architecture proposed here is that this coherent displacement can be conveniently achieved with the same Rydberg-atomic waveguide used for phonon state measurement. To this end, the atomic dipole transition has to be strongly driven in region $\mathrm{C}$. Under appropriate conditions (see Appendix C), this leads to the effective emergence of a coherent drive for the oscillator. The evolution operator describing the reduced dynamics of 

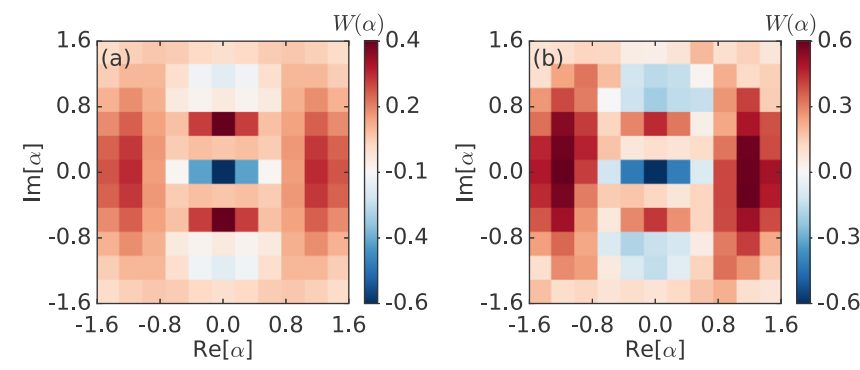

FIG. 3. Quantum state tomography of oscillator state using QNDdetection sequences and coherent displacements all with the same atomic beam. (a) Ideal Wigner function of the oscillator in state $|\Psi\rangle=$ $[|1\rangle+|3\rangle] / \sqrt{2}$. (b) Reconstruction including practical imperfections as discussed in the text.

the oscillator for a succession of $N$ atoms reads as $\hat{\mathcal{U}}_{N}(\tau)=$ $\hat{\mathcal{D}}\left[\alpha_{N}(\tau)\right] \exp \left[-i N \theta(\tau) \hat{c}^{\dagger} \hat{c}\right]$, a product of a displacement with complex amplitude $\alpha_{N}(\tau)$ depending on $\Omega$ and $\delta$, as well as a phase shift with $\theta(\tau)=\int^{\tau} d t \mathcal{K}^{2}[\mathbf{R}(t)] / \delta$ that can be compensated (see Appendix $C$ for details). Figure 2(b) shows an exemplary oscillator Wigner function before, and after, a sequence of $N=8$ displacement atoms, modeled explicitly as in the previous section. To sample the entire Wigner function with displacements of this kind, one can vary the amplitude and complex phase of the effective Rabi frequency through parameters of the external drive in region $\mathrm{C}$ (see Appendix C).

To assess the impact of the decoherence sources and imperfections mentioned earlier, we now simulate the complete Wigner tomography sequence:

(i) Initialize the oscillator in the state $\hat{\varrho}$ to be measured. This initialization must be reproducible.

(ii) Effectuate a coherent displacement $\hat{\varrho} \mapsto \hat{\varrho}(-\alpha)$, using a flyby sequence of $N$ explicitly modeled displacement atoms.

(iii) Measure the phonon number with a flyby sequence of $K$-atomic Ramsey interference measurements. The first few atoms collapse the oscillator into a Fock state $\left|n^{\prime}\right\rangle$, which is read out by the remaining majority of the $K$ atoms.

(iv) Repeat steps (i)-(iii) $N_{\mathrm{s}}$ times to obtain the phonon probability distribution $p_{n}$ for the displacement $-\alpha$.

(v) Repeat step (iv) for an $(S \times S)$ array of different values for $\alpha \in \mathbb{C}$ to obtain the Wigner function $W(\alpha)$.

Details on how we implement measurements in our simulation can be found in Appendix E. The Wigner functions reconstructed with this sequence and the ideal expectation are shown in Fig. 3 for the superposition of Fock states $|\Psi\rangle=[|1\rangle+|3\rangle] / \sqrt{2}$. It can be seen that all major qualitative features of the Wigner function, particularly the nonclassical negativity, are correctly inferred. Quantitative deviations indicate that the decoherence rates employed here should not be exceeded.

\section{CONCLUSIONS}

By porting technologies from cavity quantum electrodynamics to nanomechanics, our scheme addresses two outstanding challenges for quantum nanomechanics, namely, phonon QND detection and quantum state tomography. Thereby, we have also described a technique for the quantum coherent state displacement of nanomechanical elements. The ingredients of the hybrid setup proposed, nanofabricated oscillators and Rydberg-atomic waveguides, can naturally coexist on the same chip surface [25], and our numerical simulations demonstrate that in combination they can be used to monitor the quantum state of nanoscale oscillators despite realistic noise and decoherence sources.

\section{ACKNOWLEDGMENTS}

We gladly acknowledge fruitful discussions with K. Hammerer, S. Singh, and A. Eisfeld, and EU financial support from the Marie Curie Initial Training Network (ITN) COHERENCE (EU-Grant No. 265031).

\section{APPENDIX A: SYSTEM HAMILTONIAN}

\section{Torsional oscillator frequency}

The resonance frequency for the harmonic motion of the torsional mechanical oscillator is assumed to be [37]

$$
\omega_{\mathrm{osc}}=\sqrt{\frac{\kappa}{I}} .
$$

Here, $I$ is the total moment of inertia with respect to the symmetry axis. It takes into account the entire assembly of ferroelectric particle and carbon nanotube that comprises the oscillator. The quantity $\kappa$ denotes the torsional spring constant of the nanotube. We consider a value of $\kappa=2.085 \times 10^{-11} \mathrm{~N} \mathrm{~m}$. For a carbon nanotube of mass $m_{\mathrm{cnt}}=8.71 \times 10^{-19} \mathrm{~kg}$ (length $\ell=$ $148.54 \mathrm{~nm}$ ) and diameter $w=75.79 \mathrm{~nm}$ with a spherical ferroelectric load of mass $m_{\mathrm{sfl}}=6.31 \times 10^{-18} \mathrm{~kg}$ and radius $r=$ $63.3 \mathrm{~nm}$ we obtain a total moment of inertia $I \approx m_{\mathrm{cnt}} w^{2} / 4+$ $2 m_{\mathrm{sfl}} r^{2} / 5 \simeq 1.126 \times 10^{-32} \mathrm{~kg}^{2} \mathrm{~m}$. This finally corresponds to a frequency $\omega_{\text {osc }} /(2 \pi) \simeq 6848.69 \mathrm{MHz}$ as specified in the main text.

\section{Atom-oscillator coupling}

The atoms passing by the oscillator experience a dipoledipole interaction

$$
\hat{H}_{\mathrm{int}}=\frac{1}{4 \pi \varepsilon_{0} R^{3}}\left[\mathbf{d}_{b a} \cdot \mathbf{d}_{\mathrm{osc}}-3\left(\mathbf{d}_{\mathrm{osc}} \cdot \mathbf{u}\right)\left(\mathbf{d}_{b a} \cdot \mathbf{u}\right)\right]\left[\hat{\sigma}_{b a}+\hat{\sigma}_{a b}\right],
$$

with the permanent dipole moment $\mathbf{d}_{\text {osc }}$ of the nanoparticle attached to the oscillator. Here, the vector $\mathbf{R}$ points from the oscillator to the atom and $\mathbf{u}=\mathbf{R} / R$ with $R=\|\mathbf{R}\|$. The direction of the atomic transition dipole moment $\mathbf{d}_{b a}$ in principle depends on the states in question. For simplicity, we choose the two atomic states such that their transition dipole moment is along the $z$ axis [48]. In this case, the interaction reduces to

$$
\begin{aligned}
\hat{H}_{\mathrm{int}}(\mathbf{R}, \varphi)= & \frac{d_{b a} d_{\mathrm{osc}}}{4 \pi \varepsilon_{0} R^{3}}\left[\left(1-3 \frac{Z^{2}}{R^{2}}\right) \cos \varphi\right. \\
& \left.-3 \frac{X Z}{R^{2}} \sin \varphi\right]\left[\hat{\sigma}_{b a}+\hat{\sigma}_{a b}\right] \\
= & V_{0}[f(\mathbf{R}) \cos \varphi-g(\mathbf{R}) \sin \varphi]\left[\hat{\sigma}_{b a}+\hat{\sigma}_{a b}\right],
\end{aligned}
$$




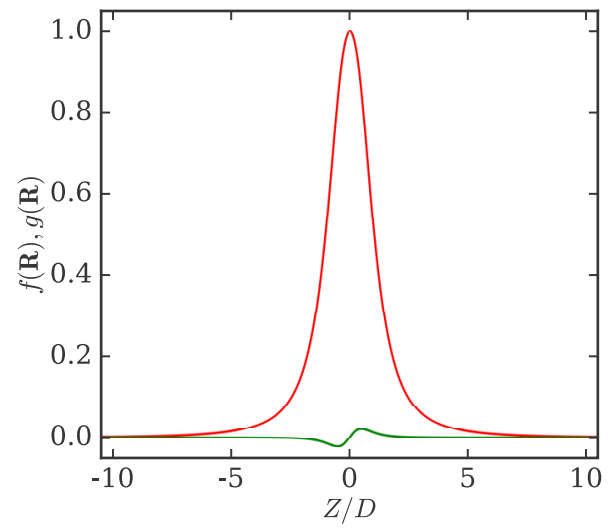

FIG. 4. Coupling functions $f(X, Y, Z)$ (red) and $g(X, Y, Z)$ (green) along the axis of the waveguide. Parameters are $D \simeq$ $21.68 \mu \mathrm{m}, X / D \simeq 0.0231$, and $Y / D=0.9997$.

with $\quad d_{\mathrm{osc}}=\left\|\mathbf{d}_{\mathrm{osc}}\right\|, d_{b a}=\left\|\mathbf{d}_{b a}\right\|, V_{0}=\frac{d_{b a} d_{\mathrm{osc}}}{4 \pi \varepsilon_{0} D^{3}}, \mathbf{R}=(X, Y, Z)$ and coupling functions $f(\mathbf{R})=[D / R]^{3}\left[1-3 Z^{2} / R^{2}\right], g(\mathbf{R})=$ $3 X Z D^{3} / R^{5}$. These are sketched for an example in Fig. 4 . We now make use of the fact that the coordinate $\varphi$ is harmonically bound to a stable equilibrium configuration given by the angle $\varphi_{0}=\pi / 2$, and introduce the small angular displacement $|\delta \varphi| \ll \varphi_{0}$, such that $\varphi=\varphi_{0}+\delta \varphi$.

Then, a Taylor series expansion of the sinusoidal functions in the potential energy, around the equilibrium value $\varphi_{0}=\pi / 2$, and up to second order in $\delta \varphi$ yields

$$
\hat{H}_{\mathrm{int}}(\mathbf{R}, \varphi) \approx V_{0}\left[g(\mathbf{R}) \delta \varphi^{2}-f(\mathbf{R}) \delta \varphi-g(\mathbf{R})\right]\left[\hat{\sigma}_{b a}+\hat{\sigma}_{a b}\right] .
$$

Next, we recast the above in terms of the mechanical phonon creation and annihilation operators $\hat{c}$ and $\hat{c}^{\dagger}$ as

$$
\delta \varphi=\varphi_{\mathrm{zpm}}\left[\hat{c}+\hat{c}^{\dagger}\right], \quad \delta \varphi^{2}=\varphi_{\mathrm{zpm}}^{2}\left[\hat{c}^{2}+\left(\hat{c}^{\dagger}\right)^{2}+2 \hat{c}^{\dagger} \hat{c}+\hat{1}_{\mathrm{osc}}\right],
$$

where $\varphi_{\mathrm{zpm}}=\sqrt{\hbar /\left(2 \omega_{\mathrm{osc}} I\right)}$ represents the amplitude of the zero point motion of the torsion oscillator and $\hat{11}_{\text {osc }}=$ $\sum_{n}|n\rangle\langle n|$.

For atoms traveling perfectly at the center of the atomic waveguide ( $X=0$, see Fig. 1 in the main text), we then obtain the interaction Hamiltonian $\hat{H}_{\text {int }}$ given in the main text.

\section{APPENDIX B: RAMSEY MEASUREMENTS OF PHASE SHIFTS}

As discussed in the main text, the train of Rydberg atoms passing the oscillator is modeled explicitly, with atom $k$ being given a randomized initial position $\mathbf{R}_{k}(0)$ and velocity $\mathbf{v}_{k}$, subsequently following a uniform trajectory $\mathbf{R}_{k}(t)=\mathbf{R}_{k}(0)+$ $\mathbf{v}_{k} t$. The initial widths of these random distributions $\sigma_{X, Y}$ in the position plane transverse to the beam and $\sigma_{v_{Z}}$ in the velocity along the beam are chosen to mimic the relevant uncertainties for a beam of atoms traveling within a very tight waveguide. Uniform motion is justified as long as forces on the atom are negligible or weak compared to waveguide trapping:
For a sufficiently large impact parameter $D$, as is the case here, the force exerted on the atoms by the electric field of the ferroelectric nanoparticle becomes negligible. If an experiment was able to reduce the transverse atomic beam velocity and position spread also without confinement, a waveguide would be dispensable and the scheme could also utilize free atomic motion.

To implement a single interferometric measurement we exert a sequence of two identical microwave pulses (mw) onto an atom. The two mw pulses are applied in $R_{1}$ and $R_{2}$, and thus they are delayed from each other by a time period $\tau=L /|\mathbf{v}|$, which is the time of flight of the atom in the region $C$ of length $L=30.872 \mu \mathrm{m}$, during which the atom is let to interact with the mechanical oscillator. We model the atomic evolution in the two locations $\mathrm{R}_{1 / 2}$ by applying a unitary transformation $\hat{A}_{\pi / 2}(\phi)$, that in the basis $\{|a\rangle,|b\rangle\}$ reads as

$$
A_{\pi / 2}(\phi)=\frac{1}{\sqrt{2}}\left(\begin{array}{cc}
1 & e^{i \phi} \\
-e^{-i \phi} & 1
\end{array}\right) .
$$

Here, $\phi$ represents a phase that in practice can be controlled by tuning the microwave pulse. In an experiment one adjusts the relative phase between the two pulses to scan the Ramsey fringes of the signals described by the probabilities $P_{b}$ and $P_{a}$ that the atom is detected in the state $|b\rangle$ or $|a\rangle$, respectively, when reaching the detector at F. We use $\hat{A}_{\pi / 2}(\phi=\pi)$ in region $\mathrm{R}_{1}$ and choose the phase of $\hat{A}_{\pi / 2}(\phi)$ in region $\mathrm{R}_{2}$ such that $P_{b}$ equals zero when the mechanical oscillator mode is in its ground state and the atom has traversed the interferometer with velocity and transverse position corresponding to the mean atomic beam values.

Finally, the relative phase shift $\Phi^{(n)}[\delta]$ incurred by each atom depends on the difference between two adjacent eigenenergies $E_{-}^{(n+1)}(\mathbf{R})-E_{+}^{(n)}(\mathbf{R})$ integrated over time and thus can be found from $E_{ \pm}^{(n)}(\mathbf{R})=\hbar \delta / 2\left[-1 \pm \sqrt{1+4 n \mathcal{K}^{2}(\mathbf{R}) / \delta^{2}}\right]$ following [27]. This helps to choose the right parameters for mapping the relative phase shift $\Phi^{(n)}[\delta]$ from a selected range of phonon numbers (e.g., $0-5)$ onto the interval $[0, \pi]$ (see Appendix F and Table I).

\section{APPENDIX C: EFFECTIVE COHERENT DRIVING OF MECHANICAL OSCILLATOR}

Simultaneous action of the Hamiltonians $\hat{H}_{\text {int }}$ and $\hat{H}_{\text {coup }}$ from the main text can effectively create a drive for the quantum harmonic oscillator. For that we need to have $|\delta|>$ $|\Omega(t)|,|\mathcal{K}(\mathbf{R})|$ and start with all the atomic population in $|a\rangle$. In this regime, we can adiabatically eliminate the second Rydberg state $|b\rangle$ to obtain the effective Hamiltonian $\hat{H}_{\text {dho }}(t) \otimes \hat{\sigma}_{a a}$, where

$$
\hat{H}_{\text {dho }}(t)=\frac{\mathcal{K}^{2}(\mathbf{R})}{\delta} \hat{c}^{\dagger} \hat{c}-\frac{\mathcal{K}(\mathbf{R}) \Omega(t)}{2 \delta} \hat{c}-\frac{\mathcal{K}(\mathbf{R}) \Omega^{*}(t)}{2 \delta} \hat{c}^{\dagger} .
$$

While the atom essentially remains in the Rydberg state $|a\rangle$, the evolution for the mechanical oscillator can be written as

$$
\hat{\varrho}(\tau)=\hat{\mathcal{U}}(\tau) \hat{\varrho}(0) \hat{\mathcal{U}}^{\dagger}(\tau),
$$

where $\hat{\varrho}$ denotes the density matrix describing the oscillator. The quantity $\hat{\mathcal{U}}(\tau)$ equals the time development operator for a driven quantum harmonic oscillator, which 
TABLE I. Parameters used for our simulations underlying Figs. 2 and 3 of the main text.

\begin{tabular}{|c|c|c|}
\hline & Symbol & Value \\
\hline \multicolumn{3}{|l|}{ Atomic system (rubidium, ${ }^{87} \mathbf{R b}$ ) } \\
\hline Mass & $M$ & $1.44 \times 10^{-25} \mathrm{~kg}$ \\
\hline $\begin{array}{l}\text { Initial atom position (with } D \text { being the } \\
\text { impact parameter) }\end{array}$ & $\mathbf{R}(t=0)=(X(0), Y(0)=D, Z(0))$ & $(0.0 \mu \mathrm{m}, 21.675 \mu \mathrm{m},-15.436 \mu \mathrm{m})$ \\
\hline Spatial atomic beam widths (transverse to $z$ axis) & $\sigma_{X}=\sigma_{Y}$ & $0.509 \mu \mathrm{m}$ \\
\hline $\begin{array}{l}\text { Initial atom velocity: displacement sequence } \\
\text { measurement sequence }\end{array}$ & $\mathbf{v}(t=0)=\left(v_{X}(0), v_{Y}(0), v_{Z}(0)\right)$ & $\begin{array}{c}\left(0 \mathrm{~m} \mathrm{~s}^{-1}, 0 \mathrm{~m} \mathrm{~s}^{-1}, 14 \mathrm{~m} \mathrm{~s}^{-1}\right) \\
\left(0 \mathrm{~m} \mathrm{~s}^{-1}, 0 \mathrm{~m} \mathrm{~s}^{-1}, 8 \mathrm{~m} \mathrm{~s}^{-1}\right)\end{array}$ \\
\hline Velocity atomic beam width (along zaxis) & $\sigma_{v_{Z}}$ & $0.01 \mathrm{~m} \mathrm{~s}^{-1}$ \\
\hline Principal quantum number & $v$ & 80 \\
\hline Rydberg state basis & $\left\{\left|v L_{J}, m_{J}\right\rangle\right\}$ & $\left\{|a\rangle=\left|80 S_{1 / 2}, 1 / 2\right\rangle,|b\rangle=\left|80 P_{1 / 2}, 1 / 2\right\rangle\right\}$ \\
\hline Transition frequency $(|b\rangle \leftrightarrow|a\rangle)$ & $\omega_{b a} /(2 \pi)$ & $6835.81 \mathrm{MHz}$ \\
\hline Electric dipole moment strength & $d_{b a}$ & $5.69 \times 10^{-26} \mathrm{Cm}$ \\
\hline \multicolumn{3}{|l|}{ Torsional mechanical oscillating mode } \\
\hline Torsional spring constant of the nanotube & $\kappa$ & $2.085 \times 10^{-11} \mathrm{Nm}$ \\
\hline $\begin{array}{l}\text { Total moment of inertia with respect to the } \\
\text { tube axis }\end{array}$ & $I$ & $1.126 \times 10^{-32} \mathrm{~kg}^{2} \mathrm{~m}$ \\
\hline $\begin{array}{l}\text { Permanent dipole moment strength of } \\
\text { ferroelectric load }\end{array}$ & $d_{\mathrm{osc}}$ & $2.58 \times 10^{-20} \mathrm{Cm}$ \\
\hline Frequency & $\omega_{\mathrm{osc}} /(2 \pi)=(2 \pi)^{-1} \sqrt{\kappa / I}$ & $6848.69 \mathrm{MHz}$ \\
\hline Number state basis & $\{|n\rangle\}$ & $\{|0\rangle,|1\rangle, \ldots,|15\rangle\}$ \\
\hline Quality factor & $Q=\omega_{\mathrm{osc}} / \Gamma_{\mathrm{osc}}$ & $1.37 \times 10^{8}$ \\
\hline Heat bath temperature & $T_{\mathrm{osc}}$ & $0.025 \mathrm{~K}$ \\
\hline
\end{tabular}

\section{Coupling and decoherence rates}

\begin{tabular}{|c|c|c|}
\hline Atom-oscillator coupling rate & $\mathcal{K}_{0} /(2 \pi)=\frac{d_{b a} d_{\text {osc }}}{8 \pi^{2} \varepsilon_{0} D^{3}} \frac{1}{\sqrt{2 \hbar \omega_{\text {osc }} I}}$ & $0.64 \mathrm{MHz}$ \\
\hline Effective Rabi frequency & $\Omega_{0} /(2 \pi)$ & $0.0 \mathrm{MHz}$ to $1.8 \mathrm{MHz}$ \\
\hline Mechanical damping rate & $\Gamma_{\text {osc }} /(2 \pi)$ & $50 \mathrm{~Hz}$ \\
\hline $\begin{array}{l}\text { Pure relaxation rate due to black-body } \\
\text { radiation induced transitions }(|b\rangle \leftrightarrow|a\rangle)\end{array}$ & $\Gamma_{\mathrm{bbr}} /(2 \pi)$ & $988.63 \mathrm{~Hz}$ \\
\hline Pure dephasing rate of $|a\rangle$ and $|b\rangle$ levels due to noisy & $\Gamma_{\text {deph }} /(2 \pi)$ & $1.50 \mathrm{kHz}$ \\
\hline
\end{tabular}

stray electric fields

\section{Protocol of state tomography}

\begin{tabular}{lcc}
\hline $\begin{array}{l}\text { Dimensions (number of pixels) of the reconstructed } \\
\text { Wigner function }\end{array}$ & $S \times S$ & $11 \times 11$ \\
$\begin{array}{l}\text { Number of atoms per displacement sequence to } \\
\text { reach a given phase space pixel }\end{array}$ & $N$ & 8 \\
$\begin{array}{l}\text { Number of atoms per measurement sequence to } \\
\text { collapse oscillator into Fock state }\end{array}$ & $N_{\mathrm{s}}$ & 43 \\
$\begin{array}{l}\text { Number of repetitions (samples) of a displacement } \\
\text { plus measurement }\end{array}$ & & 512 \\
sequence to obtain a set of phonon probabilities & $|\delta| /(2 \pi)=\left|\omega_{\text {osc }}-\omega_{b a}\right| /(2 \pi)$ & $\tau_{\text {disp }}$ \\
at a given pixel & $\tau_{\text {meas }}$ & $12.88 \mathrm{MHz}$ \\
Atom-oscillator detuning & $2.205 \mu \mathrm{s}$ \\
Passage time per atom in a displacement sequence & $3.859 \mu \mathrm{s}$ \\
Passage time per atom in a measurement sequence &
\end{tabular}

one can see by exploiting the commutation relations of $\hat{c}, \hat{c}^{\dagger}[49]$ :

$$
\begin{aligned}
& \hat{\mathcal{U}}(\tau)=e^{i \lambda(\tau)} \hat{\mathcal{D}}\left[\xi(\tau) e^{-i \theta(\tau)}\right] e^{-i \theta(\tau) \hat{c}^{\dagger} \hat{c}} \\
& \lambda(\tau)=-\frac{1}{2 \hbar^{2}} \int^{\tau} d t \int^{t} d t^{\prime}\left[\hat{H}_{\mathrm{dho}}(t), \hat{H}_{\mathrm{dho}}\left(t^{\prime}\right)\right], \\
& \xi(\tau)=\mathrm{i} \int^{\tau} d t \frac{\Omega^{*}(t) \mathcal{K}(t)}{2 \delta} e^{i \theta(t)} \\
& \theta(\tau)=\int^{\tau} d t \frac{\mathcal{K}^{2}(t)}{\delta}
\end{aligned}
$$

Here, we adopted the shortened notation $\mathcal{K}(t)=\mathcal{K}[\mathbf{R}(t)]$ and $\hat{\mathcal{D}}$ is the displacement operator introduced in the main text. Since $\lambda(\tau)$ is a $c$ number, $\exp [i \lambda(\tau)]$ is a global phasor that we will ignore from now on. The $N$ th power of $\hat{\mathcal{U}}(\tau)$ then accounts for the state evolution of the mechanical oscillator after a successive flyby of $N$ atoms, each atom passing through the oscillator in a time interval $\tau$. To compute $\hat{\mathcal{U}}_{N}(\tau) \equiv[\hat{\mathcal{U}}(\tau)]^{N}$ we use the following properties of the displacement operator:

$$
\begin{aligned}
\hat{\mathcal{D}}(\alpha) \hat{\mathcal{D}}(\beta) & =\exp \left[\left(\alpha \beta^{*}-\alpha^{*} \beta\right) / 2\right] \hat{\mathcal{D}}(\alpha+\beta), \\
\exp \left[i \theta \hat{c}^{\dagger} \hat{c}\right] \hat{\mathcal{D}}(\alpha) & =\hat{\mathcal{D}}(\alpha \exp [i \theta]) \exp \left[i \theta \hat{c}^{\dagger} \hat{c}\right],
\end{aligned}
$$


such that, ignoring again global phasors, one has

$$
\begin{aligned}
\hat{\mathcal{U}}_{N}(\tau) & =\hat{\mathcal{D}}\left[\xi(\tau) \sum_{l=1}^{N} e^{-i l \theta(\tau)}\right] e^{-i N \theta(\tau) \hat{c}^{\dagger} \hat{c}} \\
& =\hat{\mathcal{D}}\left[\alpha_{N}(\tau)\right] e^{-i N \theta(\tau) \hat{c}^{\dagger} \hat{c}} \\
\alpha_{N}(\tau) & =\frac{\sin [N \theta(\tau) / 2]}{\sin [\theta(\tau) / 2]} \xi(\tau) e^{-i[N+1] \theta(\tau) / 2} .
\end{aligned}
$$

In the paper, all the numerical calculations involving the Hamiltonian $\hat{H}_{\text {coup }}$ were assuming continuous waves with $\Omega(t)=\Omega_{0}$. Sampling the dynamical phase space of the mechanical oscillator is then achieved by adjusting the amplitude and phase of the complex Rabi frequency $\Omega_{0}$, while taking into account the additional phase offset generated by $\exp \left[-i N \theta(\tau) \hat{c}^{\dagger} \hat{c}\right]$.

\section{APPENDIX D: DECOHERENCE SOURCES}

As discussed in the main text, we consider a variety of practically relevant decoherence sources, in order to explore the limitation of our proposal. The specific Lindblad operators with which we describe the effects listed in the main text are as follows: (i) Mechanical oscillator states decohere because they are coupled to a heat bath equilibrated at a temperature $T_{\mathrm{osc}}$. This is described by the two terms $\hat{L}_{-}=\sqrt{\left(\bar{n}_{\text {th }}+1\right) \Gamma_{\text {osc }}} \hat{c}$ and $\hat{L}_{+}=\sqrt{\bar{n}_{\mathrm{th}} \Gamma_{\mathrm{osc}}} \hat{c}^{\dagger}$, with a thermal occupation number $\bar{n}_{\mathrm{th}}=$ $\left\{\exp \left[\hbar \omega_{\mathrm{osc}} /\left(k_{\mathrm{B}} T_{\mathrm{osc}}\right)\right]-1\right\}^{-1}$ and a mechanical energy damping rate $\Gamma_{\mathrm{osc}}=\omega_{\mathrm{osc}} / Q_{\mathrm{osc}}$ for a given quality factor $Q_{\mathrm{osc}}$ of the mechanical oscillator. (ii) Atomic Rydberg states are assumed to undergo pure relaxation due to black-body radiation induced transitions, modeled with two terms $\hat{L}_{\mu^{\prime} \mu}=\sqrt{\Gamma_{\mathrm{bbr}}} \hat{\sigma}_{\mu^{\prime} \mu}$, where $\left\{\mu^{\prime}, \mu\right\}=\{a, b\}$ or $\{b, a\}$. We employ $\Gamma_{\mathrm{bbr}} /(2 \pi)=988.63 \mathrm{~Hz}$, determined following [50]. (iii) They are also assumed subject to dephasing with $\hat{L}_{\mathrm{deph}, \mu}=\sqrt{\Gamma_{\mathrm{deph}}} \hat{\sigma}_{\mu \mu}$ using $\mu \in\{a, b\}$ due to stray electric fields from the oscillator-bearing surface [22]. We employ $\Gamma_{\text {deph }} /(2 \pi)=1.50 \mathrm{kHz}$, the same order of magnitude as values reported in [24].

Due to the short distances between Rydberg probes and mechanical oscillator, a primary challenge for our scheme are the finite widths of the atomic beam within the waveguide. The implementation of the interrogating atomic beam in the waveguide via a random distribution of initial positions $\mathbf{R}_{k}(0)$ and velocities $\mathbf{v}_{k}$ was discussed in Appendix B.

\section{APPENDIX E: QUANTUM STATE TOMOGRAPHY SIMULATIONS}

The physical sequence for a full oscillator quantum state tomography has been discussed in the main text. Here, we supply further technical details about the simulations. In the following, let $\hat{\rho}$ denote the full density matrix of the compound atom-oscillator system. We then use $\hat{\varrho}=\operatorname{tr}_{\mathrm{at}}[\hat{\rho}]$ for the reduced density operator of the oscillator after tracing over the atomic degrees of freedom.

Each atom, whether for a Ramsey measurement or a phase space displacement of the oscillator state, is initialized in $|a\rangle$ and made to move on a trajectory as discussed in Appendix B. Then, its flyby past the oscillator is modeled with the master equation (3) of the main text. The mw pulses required for Ramsey measurements are emulated via the instantaneous application of the operator $\hat{A}_{\pi / 2}(\phi)$ in Eq. (B1). The Lindblad terms listed in Appendix D take into account decoherence processes. Of crucial importance for modeling the experimental sequence is the final atom state detection at F. We assume detection can only yield the two states $|a\rangle$ or $|b\rangle$. To numerically represent this measurement, we compare a pseudorandom number $\eta$, drawn from a standard uniform distribution, with the probability $P_{b}=\operatorname{tr}\left[\hat{\sigma}_{b b} \hat{\rho}\right]$ that the atom is found in $b$. The output of the measurement is $\hat{\sigma}_{a a} \hat{\rho} \hat{\sigma}_{a a} /[1-$ $P_{b}$ ] if $P_{b}<\eta$ and $\hat{\sigma}_{b b} \hat{\rho} \hat{\sigma}_{b b} / P_{b}$ otherwise, thus collapsing the state onto $|a\rangle$ or $|b\rangle$ in the subspace of the atom [51]. After a series of $K=43$ atoms, typically all but one of the phonon probabilities are depopulated, such that $\varrho_{n n} \simeq \delta_{n m}$, with $\delta_{n m}$ being the Kronecker delta and $m$ a positive integer, the final phonon number. The mechanical oscillator is then assumed to have collapsed into the a priori unknown Fock state $|m\rangle$. In the theory, we can directly extract $m$ from the simulation, repeat the process multiple times, and thus extract the entire phonon distribution. We call this approach "Method A".

However, an experiment would not have access to $m$ directly, instead it would extract the probabilities $P_{a / b}$ from the measurement results of the $K=43$ probing atoms. Using Fig. 2(a) of the main text, these can then be translated into values of $m$, but this translation may be subject to different error sources. We also extract a second value of $m$ from the simulation in this manner, called "Method B".

We finally sample the entire phase space of the oscillator on a square grid of $S \times S$ points, by explicitly modeling different displacements $\alpha \in \mathbb{C}$. The density matrix is propagated in time using Eq. (3) of the main text, while the passing atoms are driven, such that it effectively evolves as described by the operator $\hat{\mathcal{U}}_{N}$. This is described in Appendix $\mathrm{C}$ and visualized in Fig. 2(b) of the main text. For each point of the square grid a phonon count distribution is sampled and the Wigner function is finally computed as discussed in the main text.

Figure 3(b) of the main text illustrates the Wigner density of the mechanical oscillator superposition state $|\Psi\rangle=[|1\rangle+$ $|3\rangle] / \sqrt{2}$ as obtained through Method A. Here, we show it again in Fig. 5(a) together with Fig. 5(b), which depicts also the Wigner density of $|\Psi\rangle=[|1\rangle+|3\rangle] / \sqrt{2}$ derived in this case from Method B. The Wigner density computed via
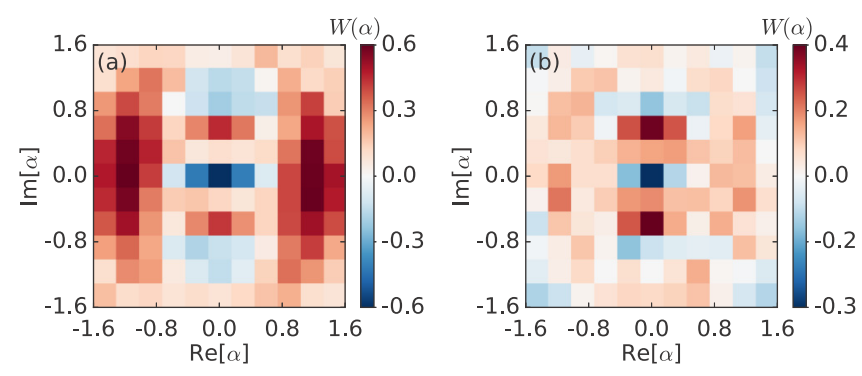

FIG. 5. Tomographical reconstruction of the Wigner density $W(\alpha)$ of the mechanical oscillator superposition state $|\Psi\rangle=[|1\rangle+$ $|3\rangle] / \sqrt{2}$. (a) Evaluation of $W(\alpha)$ using the reconstruction protocol of Method A. (b) The outcome of the same $W(\alpha)$ applying instead the protocol of Method B. Both Methods A and B are defined in Appendix E. 
Method A resembles the exact outcome displayed in Fig. 3(a) of the main text more than the counterpart result determined through Method B. Considering that occasionally a series of $K=43$ Ramsey measurements may not suffice to project the mechanical oscillator state into a Fock state, we expect a higher inaccuracy of Method B compared to Method A. Indeed, if a complete Fock state collapse is not realized, applying Method B may lead to a wrong Fock state record or to the loss of a statistical sample. Contrarily, if we apply Method B in a similar situation, a statistical loss never occurs and the error for a Fock state miscount is lower.

\section{APPENDIX F: SYSTEM DESIGN AND PARAMETER CHOICES}

The following central requirements dictate the choice of parameters for the setup: (i) The distance $L$ between regions $\mathrm{R}_{1,2}$ and the parameters $D$ and $\mathbf{v}$ have to be adjusted such that (a) the average flyby time $\tau$ of an atom across the interferometer is much shorter than the Rydberg lifetime, and (b) each atomic record can serve as a nondestructive measurement of discrete (phonon-) number states in the range between $n=0$ and 5, as can be seen from the atomic excited-state probability $P_{b}$ shown in Fig. 2(a) of the main text.

(ii) For each detection event there is no energy exchange between the atom and the mechanical oscillator. In other words, the instantaneous atom-oscillator coupling is designed to remain sufficiently weak (off resonant) until the atom interferometric measurement is completed. In this way, transitions between atomic states $|b\rangle$ and $|a\rangle$ via the absorption or emission of a phonon are negligible, such that the mechanical oscillator contains $n$ phonons before and after the atom traverses the interferometer.
[1] B. P. Abbott, R. Abbott, T. D. Abbott, M. R. Abernathy, F. Acernese, K. Ackley, C. Adams, T. Adams, P. Addesso, R. X. Adhikari et al. (LIGO Scientific Collaboration and Virgo Collaboration), Phys. Rev. Lett. 116, 061102 (2016).

[2] J. Chan, T. P. M. Alegre, A. H. Safavi-Naeini, J. T. Hill, A. Krause, S. Gröblacher, M. Aspelmeyer, and O. Painter, Nature (London) 478, 89 (2011).

[3] M. Aspelmeyer, T. J. Kippenberg, and F. Marquardt, Rev. Mod. Phys. 86, 1391 (2014).

[4] T. Kippenberg and K. Vahala, Opt. Express 15, 17172 (2007).

[5] M. Poot and H. S. van der Zant, Phys. Rep. 511, 273 (2012).

[6] T. A. Palomaki, J. D. Teufel, R. W. Simmonds, and K. W. Lehnert, Science 342, 710 (2013).

[7] R. Riedinger, S. Hong, R. A. Norte, J. A. Slater, J. Shang, A. G. Krause, V. Anant, M. Aspelmeyer, and S. Gröblacher, Nature (London) 530, 313 (2016).

[8] F. Lecocq, J. B. Clark, R. W. Simmonds, J. Aumentado, and J. D. Teufel, Phys. Rev. X 5, 041037 (2015).

[9] C. U. Lei, A. J. Weinstein, J. Suh, E. E. Wollman, A. Kronwald, F. Marquardt, A. A. Clerk, and K. C. Schwab, Phys. Rev. Lett. 117, 100801 (2016).

[10] M. R. Vanner, I. Pikovski, and M. S. Kim, Ann. Phys. (NY) 527, 15 (2015).

[11] M. J. Woolley, A. C. Doherty, and G. J. Milburn, Phys. Rev. B 82, 094511 (2010).

[12] A. A. Gangat, T. M. Stace, and G. J. Milburn, New J. Phys. 13, 043024 (2011).

[13] M. Ludwig, A. H. Safavi-Naeini, O. Painter, and F. Marquardt, Phys. Rev. Lett. 109, 063601 (2012).

[14] Z.-q. Yin, T. Li, X. Zhang, and L. M. Duan, Phys. Rev. A 88, 033614 (2013).

[15] P. H. Kim, B. D. Hauer, C. Doolin, F. Souris, and J. P. Davis, Nat. Commun. 7, 13165 (2016).

[16] P. H. Kim, C. Doolin, B. D. Hauer, A. J. R. MacDonald, M. R. Freeman, P. E. Barclay, and J. P. Davis, Appl. Phys. Lett. 102, 053102 (2013).

[17] T. F. Gallagher, Rydberg Atoms (Cambridge University Press, Cambridge, 1994).
[18] S. D. Hogan, P. Allmendinger, H. Saßmannshausen, H. Schmutz, and F. Merkt, Phys. Rev. Lett. 108, 063008 (2012).

[19] P. Lancuba and S. D. Hogan, Phys. Rev. A 88, 043427 (2013).

[20] P. Lancuba and S. D. Hogan, Phys. Rev. A 90, 053420 (2014).

[21] J. D. Carter, O. Cherry, and J. D. D. Martin, Phys. Rev. A 86, 053401 (2012).

[22] J. D. Carter and J. D. D. Martin, Phys. Rev. A 88, 043429 (2013).

[23] T. Thiele, S. Filipp, J. A. Agner, H. Schmutz, J. Deiglmayr, M. Stammeier, P. Allmendinger, F. Merkt, and A. Wallraff, Phys. Rev. A 90, 013414 (2014).

[24] C. Hermann-Avigliano, R. C. Teixeira, T. L. Nguyen, T. CantatMoltrecht, G. Nogues, I. Dotsenko, S. Gleyzes, J. M. Raimond, S. Haroche, and M. Brune, Phys. Rev. A 90, 040502(R) (2014).

[25] M. Keil, O. Amit, S. Zhou, D. Groswasser, Y. Japha, and R. Folman, J. Mod. Opt. 63, 1840 (2016).

[26] S. D. Hogan, Eur. Phys. J.: Techniques Instrum. 3, 2 (2016).

[27] M. Brune, S. Haroche, V. Lefevre, J. M. Raimond, and N. Zagury, Phys. Rev. Lett. 65, 976 (1990).

[28] M. Brune, J. Bernu, C. Guerlin, S. Deléglise, C. Sayrin, S. Gleyzes, S. Kuhr, I. Dotsenko, J. M. Raimond, and S. Haroche, Phys. Rev. Lett. 101, 240402 (2008).

[29] M. Brune, S. Haroche, J. M. Raimond, L. Davidovich, and N. Zagury, Phys. Rev. A 45, 5193 (1992).

[30] S. Gleyzes, S. Kuhr, C. Guerlin, J. Bernu, S. Deléglise, U. B. Hoff, M. Brune, J.-M. Raimond, and S. Haroche, Nature (London) 446, 297 (2007).

[31] S. Deléglise, I. Dotsenko, C. Sayrin, J. Bernu, M. Brune, J.-M. Raimond, and S. Haroche, Nature (London) 455, 510 (2008).

[32] S. Singh and P. Meystre, Phys. Rev. A 81, 041804(R) (2010).

[33] S. Singh, M. Bhattacharya, O. Dutta, and P. Meystre, Phys. Rev. Lett. 101, 263603 (2008).

[34] F. Bariani, J. Otterbach, H. Tan, and P. Meystre, Phys. Rev. A 89, 011801(R) (2014).

[35] R. Stevenson, J. Minář, S. Hofferberth, and I. Lesanovsky, Phys. Rev. A 94, 043813 (2016).

[36] M. Gao, Y.-x. Liu, and X.-B. Wang, Phys. Rev. A 83, 022309 (2011).

[37] J. Meyer, M. Paillet, and S. Roth, Science 309, 1539 (2005). 
[38] M. Ganzhorn, S. Klyatskaya, M. Ruben, and W. Wernsdorfer, Nat. Nanotechnol. 8, 165 (2013).

[39] S. A. Basun, G. Cook, V. Y. Reshetnyak, A. V. Glushchenko, and D. R. Evans, Phys. Rev. B 84, 024105 (2011).

[40] S. D. Hogan, J. A. Agner, F. Merkt, T. Thiele, S. Filipp, and A. Wallraff, Phys. Rev. Lett. 108, 063004 (2012).

[41] This $\pi / 2$ pulse could be achieved without additional microwave cavities, but microwave fields should not directly affect the oscillator.

[42] C. Guerlin, J. Bernu, S. Deléglise, C. Sayrin, S. Gleyzes, S. Kuhr, M. Brune, J.-M. Raimond, and S. Haroche, Nature (London) 448, 889 (2007).

[43] M. A. Beck, J. A. Isaacs, D. Booth, J. D. Pritchard, M. Saffman, and R. McDermott, Appl. Phys. Lett. 109, 092602 (2016).

[44] T. Thiele, J. Deiglmayr, M. Stammeier, J.-A. Agner, H. Schmutz, F. Merkt, and A. Wallraff, Phys. Rev. A 92, 063425 (2015).
[45] N. R. de Melo and S. S. Vianna, Phys. Rev. A 92, 053830 (2015).

[46] S. Haroche and J.-M. Raimond, Exploring the Quantum: Atoms, Cavities, and Photons (Oxford University Press, Oxford, 2006).

[47] These rather low oscillator decoherence rates are required by the slow measurement chosen here. Higher values could be dealt with by a higher launching rate of atoms into the interferometer.

[48] We can for example define a quantization axis $\| z$ through Rydberg excitation laser polarization and work with $m_{j}=\frac{1}{2}$ states only.

[49] C. Gardiner and P. Zoller, Quantum Noise: A Handbook of Markovian and Non-Markovian Quantum Stochastic Methods with Applications to Quantum Optics (Springer, Berlin, 2004), Vol. 56.

[50] I. I. Beterov, I. I. Ryabtsev, D. B. Tretyakov, and V. M. Entin, Phys. Rev. A 79, 052504 (2009).

[51] H. M. Wiseman and G. J. Milburn, Quantum Measurement and Control (Cambridge University Press, Cambridge, 2009). 